\title{
Multi-Degree Reduction of Bézier Curves with Distance and Energy Optimization
}

\author{
Xuli Han, Jing Yang \\ Department of Mathematics and Statistics, Central South University, Changsha, China \\ Email: xlhan@csu.edu.cn, jane_young@csu.edu.cn
}

Received 11 November 2015; accepted 5 January 2016; published 12 January 2016

\begin{abstract}
In this paper, we propose a new approach to the problem of degree reduction of Bézier curves based on the given endpoint constraints. A differential term is added for the purpose of controlling the smoothness to a certain extent. Considering the adjustment of second derivative in curve design, a modified objective function including two parts is constructed here. One part is a kind of measure of the distance between original high order Bézier curve and degree-reduced curve. The other part represents the second derivative of degree-reduced curve. We tackle two kinds of conditions which are position vector constraint and tangent vector constraint respectively. The explicit representations of unknown points are presented. Some examples are illustrated to show the influence of the differential terms to approximation and smoothness effect.
\end{abstract}

\section{Keywords}

Bézier Curve, Degree Reduction, Endpoint Constraint, Differential Constraint, $L_{2}$-Norm

\section{Introduction}

Degree reduction of Bézier curves is an important and classical problem in CAGD (Computer Aided Geometric Design). It is to approximate the given curve with a Bézier curve of a lower degree while the approximation error is minimized. Degree reduction of curves is needed for the convenience of data exchange and transmission. It is frequently used in data compression as well. Besides, it is also useful for computing roots of polynomials [1].

Many researches dealing with this problem have been done in recent years. These researches can be classified by norm which the distance between polynomials is measured in, such as $L_{\infty}$-norm [2] [3], $L_{1}$-norm [4], $L_{2}$ -norm [5] [8], or $L_{p}$-norm [9] [10]. And the constrained degree reduction of Bézier curves with different parametric [6] [11] and geometric [5] [7] [12] continuity attend points have been studied in many previous papers.

The modification of conventional optimal function has also become a research hotspot recently. Lu [12] presented a novel approach to consider the multi-degree reduction of Bézier curves with $G^{2}$-continuity in $L_{2}$-norm, and the modification optimal approximation is obtained by minimizing the objective function based on the $L_{2}$-error between the two curves. For solving the similar degree reduction problem, Przemysław [13] impose restrictions of the control point area to get more intuitive location of the control points. Xu [14] used the method of energy-minimizing to construct curves. 
In this paper, we add a differential item which is the second derivative of the degree-reduced curve based on the conventional optimal function. It is well known that second derivative of a curve plays a leading role in curvature decision. In other words, second derivative can reflect curvature to a great extent. What is more, curvature and smoothness are closely linked and a sudden change of curvature may influence the smoothness of the curve. So the smoothness can be controlled to a certain extent by using the additional term. The distance part and the differential part are combined with a weight $\omega$ here. $L_{2}$-norm is taken to measure the distance between the degree-reduced curve and the given one. It turns to be the conventional optimal function when $\omega=0$ and $\omega=$ 1 represents square of the norm of second derivative of the degree reduced curve.

\section{Description about Degree Reduction of Bézier Curves}

In this paper, $\|\cdot\|$ denotes the Euclidean norm which means that $v=\sqrt{v_{1}^{2}+v_{2}^{2}+\cdots+v_{n}^{2}}$ for a vector $\boldsymbol{v}=\left[v_{1}, v_{2}, \cdots, v_{n}\right]$. Let $\Pi_{n}^{\alpha}(\alpha=2,3 ; n \in N)$ denote the space of all parametric polynomials in $\mathbb{R}^{\alpha}(\alpha=2,3)$ of a degree at most $n$. Let the vector $\boldsymbol{p}:=\left[\boldsymbol{p}_{0}, \boldsymbol{p}_{1}, \cdots, \boldsymbol{p}_{n}\right], \boldsymbol{p}_{i} \in \mathbb{R}^{\alpha}(i=0,1, \cdots, n)$ be control points of Bézier curve $\boldsymbol{P}_{n} \in \Pi_{n}^{\alpha}$ in the following form,

$$
\boldsymbol{P}_{n}(t)=\sum_{i=0}^{n} \boldsymbol{p}_{i} B_{i}^{n}(t),(0 \leq t \leq 1)
$$

where $B_{i}^{n}$ denote the Bernstein polynomials given by $B_{i}^{n}(t)=\left(\begin{array}{c}n \\ i\end{array}\right) t^{i}(1-t)^{n-i},(i=0,1, \cdots, n)$. The product of two Bernstein polynomials is given by

$$
B_{i}^{n}(t) B_{j}^{m}(t)=\frac{\left(\begin{array}{c}
n \\
i
\end{array}\right)\left(\begin{array}{c}
m \\
j
\end{array}\right)}{\left(\begin{array}{c}
n+m \\
i+j
\end{array}\right)} B_{i+j}^{n+m}(t) .
$$

The integral of a Bernstein polynomial is

$$
\int_{0}^{1} B_{i}^{n}(t) d t=\frac{1}{n+1}
$$

and the derivative is

$$
\left(B_{i}^{n}(t)\right)^{\prime}=n B_{i-1}^{n-1}(t)+n B_{i}^{n-1}(t) .
$$

In this paper, we define a $(n+1) \times(m+1)$-matrix $M_{n, m}$, whose elements are the integrals of products of Bernstein polynomials as follows:

$$
m_{i, j}^{n, m}=\int_{0}^{1} B_{i}^{n}(t) B_{j}^{m}(t) d t=\frac{\left(\begin{array}{c}
n \\
i
\end{array}\right)\left(\begin{array}{c}
m \\
j
\end{array}\right)}{(n+m+1)\left(\begin{array}{c}
n+m \\
i+j
\end{array}\right)}
$$

The matrix $M_{n, m}$ is real, symmetric and positive definite, which had been proved in [13].

The forward differential operator $\Delta$ is defined as follows,

$$
\Delta^{k+1} \boldsymbol{p}_{i}=\Delta^{k} \boldsymbol{p}_{i+1}-\Delta^{k} \boldsymbol{p}_{i}, \Delta^{0} \boldsymbol{p}_{i}=\boldsymbol{p}_{i},
$$

where $k \geq 0, i=0,1, \cdots, n-k-1$. The $k$-th derivatives of the Bézier curve expressed in Equation (1) at endpoints are given by

$$
\boldsymbol{P}_{n}^{(k)}(0)=\frac{n !}{(n-k) !} \Delta^{k} \boldsymbol{p}_{0}, \boldsymbol{P}_{n}^{(k)}(1)=\frac{n !}{(n-k) !} \Delta^{k} \boldsymbol{p}_{n-k} .
$$


The problem we are considering is to find a Bézier curve of degree $m$

$$
\boldsymbol{R}_{m}(t)=\sum_{j=0}^{m} \boldsymbol{r}_{j} B_{j}^{m}(t),(0 \leq t \leq 1),
$$

which is the multi-degree reduction curve of $\boldsymbol{P}_{n}$ and $m<n$. Let the vector $\boldsymbol{r}:=\left[\boldsymbol{r}_{0}, \boldsymbol{r}_{1}, \cdots, \boldsymbol{r}_{m}\right]$ be control points and $\boldsymbol{r}_{j} \in \mathbb{R}^{\alpha}(j=0,1, \cdots, m)$ of Bézier curve $\boldsymbol{R}_{m}$.

In previous researches, least square error which represents the distance between the given Bézier curve and degree-reduced curve is always taken as an optimal function [5] [13] [14], then the optimal problem can be:

$$
\min \int_{0}^{1}\left\|\boldsymbol{P}_{n}(t)-\boldsymbol{R}_{m}(t)^{2}\right\| d t .
$$

Besides, there are some continuity constraint conditions attend points. [6] [8] and [12] are mainly about $C^{r}$ and $C^{s}$-continuity at different endpoints, $r$ and $s(r, s=0,1)$ are the orders of continuity at $t=0$ and $t=1$ respectively. Multi-degree reduction of Bézier curves with $G^{1}, G^{2}$ and $G^{3}$-continuity at the endpoints of the curve are derived in [5]. Two types of geometric constraints are presented in [7]. One is $G^{1}$ and $G^{2}$-continuity at different endpoints and the other one is $G^{2}$-continuity at both sides.

There are however some other factors needed to consider in curve design, such as smoothness or energy. Note that in this paper, as second derivative has decisive effect on curvature, we add a differential term which is the second derivative of degree-reduced curve based on the conventional optimal function mentioned above. So the smoothness or energy can be controlled to a certain extent by using the differential term. What is more, for example, the process of adjusting this term becomes useful when global or local acceleration in movement needs to be lowered so that the speed variation can be more uniform in certain part.

For the convenience of adjusting the smoothness degree of degree-reduced curve, we denote an objective function

$$
\boldsymbol{F}\left(\boldsymbol{r}_{0}, \boldsymbol{r}_{1}, \cdots, \boldsymbol{r}_{m}\right):=\frac{\omega}{m(m-1)} \int_{0}^{1}\left\|\boldsymbol{R}_{m}^{\prime \prime}(t)\right\|^{2} d t+(1-\omega) \int_{0}^{1}\left\|\boldsymbol{P}_{n}(t)-\boldsymbol{R}_{m}(t)\right\|^{2} d t
$$

with a weight $\omega(0 \leq \omega \leq 1)$ as the modified objective function. Adding the denominator $m(m-1)$ in the first term is designed to keep the consistency of operation.

To solve the unknown control points of $\boldsymbol{R}_{m}$, a minimization of Equation (7) is required:

$$
\min \boldsymbol{F}\left(\boldsymbol{r}_{0}, \boldsymbol{r}_{1}, \cdots, \boldsymbol{r}_{m}\right) \text {. }
$$

It is obvious that Equation (8) turns to the conventional optimal problem Equation (6) when $\omega=0$.

\section{Degree Reduction with Endpoint Constraints}

In this section, the explicit matrix expressions of unknown points are given. Two kinds of parametric continuity are taken into consideration. For $\mathrm{C}^{0}$ and $\mathrm{C}^{1}$ continuity constraints mentioned above, considering the meaning of continuity, we would like to rename it endpoint constraints in the following. One is position vector constraint, the other one is tangent vector constraint.

Firstly, we simplify the derivative part of the modified objective function in the following by using Equation (2) and Equation (3),

$$
\frac{\omega}{m(m-1)} \int_{0}^{1}\left\|\boldsymbol{R}_{m}^{\prime \prime}(t)\right\|^{2} d t=\omega \boldsymbol{r} A^{T} M_{m-2, m-2} A \boldsymbol{r}^{T},
$$

where

$$
A:=\left[\begin{array}{cccccc}
1 & 2 & 1 & & & * \\
& 1 & 2 & 1 & & \\
& & \ddots & \ddots & \ddots & \\
* & & 1 & 2 & 1
\end{array}\right]_{(m-1) \times(m+1)}
$$


and $M_{m-2, m-2}=\left(m_{i, j}^{m-2, m-2}\right)$ is a $(m-1) \times(m-1)$ matrix described in Equation (3).

For the second part of the modified optimal function, notice that

$$
\int_{0}^{1}\left\|\boldsymbol{P}_{n}(t)-\boldsymbol{R}_{m}(t)\right\|^{2} d t=\int_{0}^{1}\left\|\boldsymbol{p} B_{n}(t)-r \boldsymbol{B}_{m}(t)\right\|^{2} d t
$$

where $\boldsymbol{B}_{n}(t)=\left[B_{0}^{n}(t), B_{1}^{n}(t), \cdots, B_{n}^{n}(t)\right]^{T}, \boldsymbol{B}_{m}(t)=\left[B_{0}^{m}(t), B_{1}^{m}(t), \cdots, B_{m}^{m}(t)\right]^{T}$. Then this part can be rewritten as:

$$
(1-\omega) \int_{0}^{1}\left\|\boldsymbol{P}_{n}(t)-\boldsymbol{R}_{m}(t)\right\|^{2} d t=(1-\omega)\left(\boldsymbol{r} M_{m, m} \boldsymbol{r}^{T}-\boldsymbol{r} M_{m, n} \boldsymbol{p}^{T}-\boldsymbol{p} M_{n, m} \boldsymbol{r}^{T}+\boldsymbol{p} M_{n, n} \boldsymbol{p}^{T}\right),
$$

where the four matrixs $M_{m, m}, M_{m, n}, M_{n, m}$ and $M_{n, m}$ are all derived from Equation (3). What is more, $M_{n, n}$ is obviously the transposition of $M_{m, n}, M_{m, m}$ and $M_{n, m}$ here are both real, symmetric and positive definite.

So for the modified optimal function $\boldsymbol{F}$, we have

$$
\boldsymbol{F}(\boldsymbol{r})=\omega \boldsymbol{r} A^{T} M_{m-2, m-2} A \boldsymbol{r}^{T}+(1-\omega)\left(\boldsymbol{r} M_{m, m} \boldsymbol{r}^{T}-2 \boldsymbol{r} M_{m, n} \boldsymbol{p}^{T}+\boldsymbol{p} M_{n, n} \boldsymbol{p}^{T}\right)
$$

\section{Position Vector Constraint}

For constraint of position at endpoints to curves $\boldsymbol{P}_{n}(t)$ and $\boldsymbol{R}_{m}(t)$, we set

$$
\boldsymbol{P}_{n}(0)=\boldsymbol{R}_{m}(0), \boldsymbol{P}_{n}(1)=\boldsymbol{R}_{m}(1),
$$

which means the points $\boldsymbol{r}_{0}$ and $\boldsymbol{r}_{m}$ in degree-reduced curve $\boldsymbol{R}_{m}(t)$ are known by Equation (4) under this situation,

$$
\boldsymbol{r}_{0}=\boldsymbol{p}_{0}, \boldsymbol{r}_{m}=\boldsymbol{p}_{n} .
$$

So there are $m-1$ points $\boldsymbol{r}_{1}, \boldsymbol{r}_{2}, \cdots, \boldsymbol{r}_{m-1}$ left to be determined. We take partial of Equation (9) respect to these unknown points to get the extremum of Equation (8), then:

$$
\omega \boldsymbol{r}^{k} A^{T} M_{m-2, m-2} A \boldsymbol{r}^{T}+(1-\omega)\left(\boldsymbol{r}^{k} M_{m, m} \boldsymbol{r}^{T}-\boldsymbol{r}^{k} M_{m, n} \boldsymbol{p}^{T}\right)=0,
$$

where $\boldsymbol{r}^{k}:=[0, \cdots, 0,1,0, \cdots, 0],(k=1,2, \cdots, m-1)$ is an unit vector and superscript $k$ means the " 1 " is at the $(k+$ 1)th position of this vector. Let $C^{k}(\omega):=\omega r^{k} A^{T} M_{m-2, m-2} A+(1-\omega) r^{k} M_{m, m}, \quad f^{k}:=r^{k} M_{m . n} P^{T}$ then Equation (11) can be simplified further as:

$$
C^{k}(\omega) \boldsymbol{r}^{T}=(1-\omega) f^{k}
$$

It is obvious that $\boldsymbol{C}^{k}(\omega)$ is a $1 \times(m+1)$ vector which can be written as $\boldsymbol{C}^{k}(\omega)=\left[C_{0}^{k}(\omega), C_{1}^{k}(\omega), \cdots, C_{m}^{k}(\omega)\right]$. Let $\overline{\boldsymbol{r}}$ be the vector of unknown points, namely $\overline{\boldsymbol{r}}:=\left[\boldsymbol{r}_{1}, \boldsymbol{r}_{2}, \cdots, \boldsymbol{r}_{m-1}\right]$, under the condition of constraints at endpoints and $\overline{\boldsymbol{C}}_{0}^{k}(\omega):=\left[C_{1}^{k}(\omega), C_{2}^{k}(\omega), \cdots, C_{m-1}^{k}(\omega)\right]$. Then we have

$$
\overline{\boldsymbol{C}}_{0}^{k}(\omega)(\overline{\boldsymbol{r}})^{T}=V_{0}^{k}(\omega), k=1,2, \cdots, m-1,
$$

where $V_{0}^{k}(\omega):=(1-\omega) f^{k}-C_{0}^{k} \boldsymbol{p}_{0}-C_{m}^{k} \boldsymbol{p}_{n}$. Thus the positions of remaining unknown points can be worked out by solving the $m-1$ equations (12).

\section{Tangent Vector Constraint}

In this section, for constraint of tangent vector at endpoints to curves $\boldsymbol{P}_{n}(t)$ and $\boldsymbol{R}_{m}(t)$, we let

$$
\begin{gathered}
\boldsymbol{P}_{n}(0)=\boldsymbol{R}_{m}(0), \boldsymbol{P}_{n}(1)=\boldsymbol{R}_{m}(1), \\
\boldsymbol{P}_{n}^{(1)}(0)=\boldsymbol{R}_{m}^{(1)}(0), \boldsymbol{P}_{n}^{(1)}(1)=\boldsymbol{R}_{m}^{(1)}(1),
\end{gathered}
$$

and we get

$$
\boldsymbol{r}_{0}=\boldsymbol{p}_{0}, \boldsymbol{r}_{m}=\boldsymbol{p}_{n}
$$




$$
\boldsymbol{r}_{1}=\frac{(m-n) \boldsymbol{p}_{0}+n \boldsymbol{p}_{1}}{m}, \boldsymbol{r}_{m-1}=\frac{(m-n) \boldsymbol{p}_{n}+n \boldsymbol{p}_{n-1}}{m} .
$$

Then the vector $\overline{\boldsymbol{r}}$ of unknown points turns to $\overline{\boldsymbol{r}}:=\left[\boldsymbol{r}_{2}, \boldsymbol{r}_{3}, \cdots, \boldsymbol{r}_{m-2}\right]$. Similar to the previous section, we take partial of Equation (9) respect to these unknown points to get the extremum of Equation (8), then we have:

$$
\overline{\boldsymbol{C}}_{1}^{k}(\omega)(\overline{\boldsymbol{r}})^{T}=V_{1}^{k}(\omega), k=2,3, \cdots, m-2,
$$

where $\overline{\boldsymbol{C}}_{1}^{k}(\omega):=\left[C_{2}^{k}(\omega), C_{3}^{k}(\omega), \cdots, C_{m-2}^{k}(\omega)\right]$ and

$$
V_{1}^{k}(\omega):=(1-\omega) f^{k}-C_{0}^{k} \boldsymbol{p}_{0}-C_{m}^{k} \boldsymbol{p}_{n}-C_{1}^{k} \frac{(m-n) \boldsymbol{p}_{0}+n \boldsymbol{p}_{1}}{m}-C_{m-1}^{k} \frac{(m-n) \boldsymbol{p}_{n}+n \boldsymbol{p}_{n-1}}{m} .
$$

Same as 3.1, the positions of remaining $m-3$ unknown points can be worked out by solving the $m-3$ Equations (14).

\section{Graphic Experiments}

We first consider a given planar Bézier curve of degree eleven $(n=11)$. Figure 1 gives the comparison of the original high order Bézier curve with degree reduced curves $(m=6)$ of different weight under constraint (10). Figure 2 shows the constraint (13). The red one is the degree reduced curve derived from the conventional optimal function of Equation (6). The green and blue ones are obtained using the modified method of Equation (8). In Table 1, $P_{s}$ represents the norm $\sqrt{\left\|\boldsymbol{P}_{n}^{\prime \prime}\right\|^{2}}, R_{s}^{1}$ represents the norm $\sqrt{\left\|\boldsymbol{R}_{m}^{\prime \prime}\right\|^{2}}$ with endpoint constraint (10) and $R_{s}^{2}$ means the same norm with endpoint constraint (13). Furthermore, $\hat{A}$ denotes the maximum of $A$, $\bar{A}$ denotes the mean of $A$ and 100 points are taken uniformly.

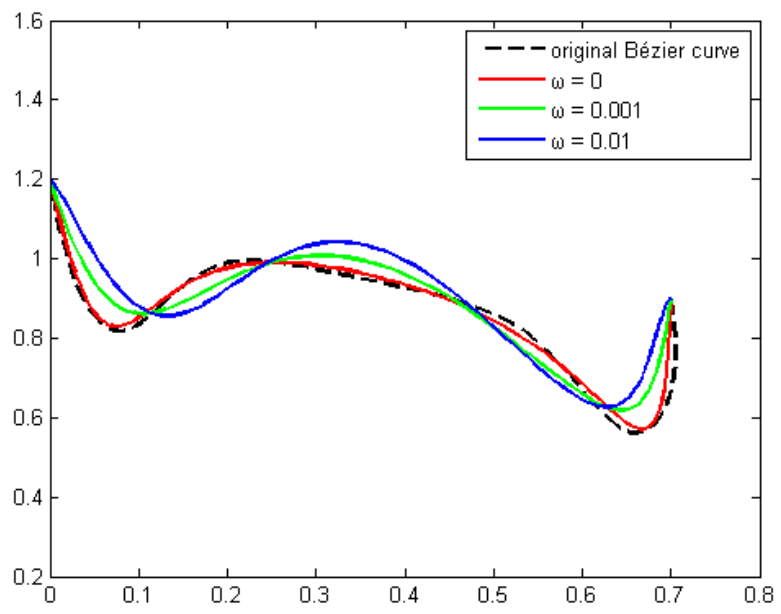

Figure 1. Degree reduced curves with constraint (10).

Table 1. Effects of different parameters to second derivative ofdegree-reduced curves in Example 1.

\begin{tabular}{cccccccc}
\hline$m$ & $\omega$ & $\hat{P}_{s}$ & $\bar{P}_{s}$ & $\hat{R}_{s}^{1}$ & $\bar{R}_{s}^{1}$ & $\hat{R}_{s}^{2}$ & $\bar{R}_{s}^{2}$ \\
\hline 6 & 0 & 61.04 & 18.97 & 118.58 & 21.40 & 84.89 & 17.57 \\
6 & 0.001 & 61.04 & 18.97 & 34.03 & 13.40 & 85.04 & 17.57 \\
5 & 0.001 & 61.04 & 18.97 & 44.15 & 13.32 & 66.71 & 19.51 \\
6 & 0.02 & 61.04 & 18.97 & 57.89 & 15.05 & 88.00 & 17.68 \\
5 & 0.02 & 61.04 & 18.97 & 8.16 & 2.82 & 67.68 & 19.76 \\
\hline
\end{tabular}




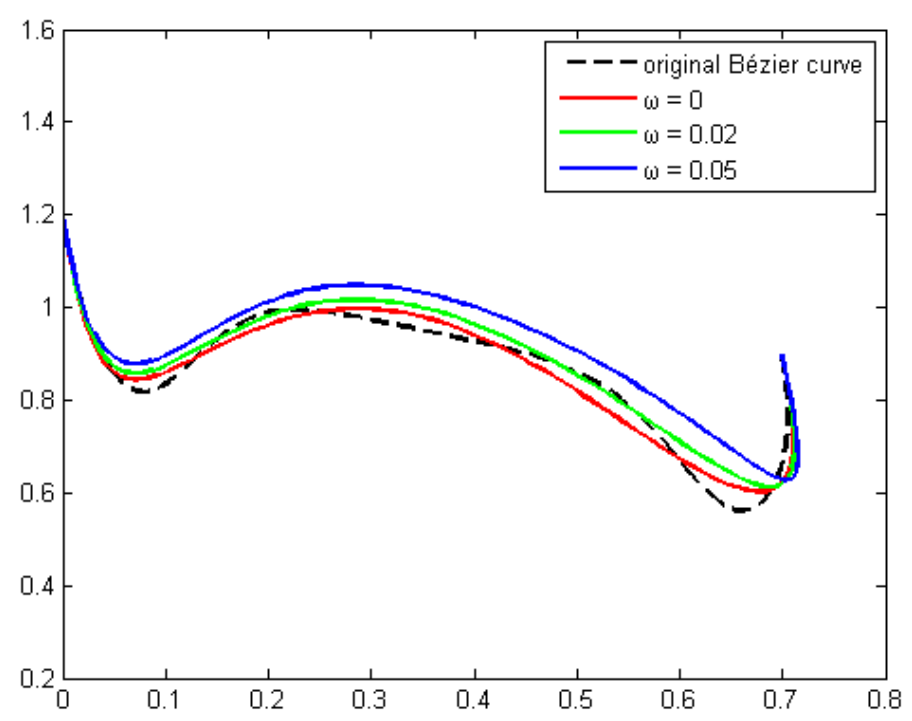

Figure 2. Degree reduced curves with constraint (13).

The maximum of second derivative of a curve can represents its smooth degree to some extent while the mean can reflect its energy. We can see that curves in Figure 1 look smoother than ones in Figure $\mathbf{2}$ which seems the smoothing term has a more remarkable effect on curves with endpoint constraint (10) and data in Table 1 proves this guess. In Figure 1, combining with the data in Table 1, increasing the weight $\omega$ of smoothing term can improve the smooth degree of degree-reduced curves based on the endpoint constraint (10). In a word, degree-reduced curves derived from the optimal function with an additional differential term are global smooth under the endpoint constraint (10), but local smooth under endpoint constraint (13) which is more restricted.

The following example is a Bézier curve of degree nineteen $(n=19)$. A degree-reduced curve with degree of seven $(m=7)$ is needed to approximate the original one. Two kinds of endpoint conditions are presented. We can see from these two figures that the trend of original Bézier curve always changes to an opposite direction. As the weight $\omega$ increases, degree-reduced curves in Figure 3 with endpoint constraint (10) become smoother and the related second derivative values become lower than ones in Figure 4, either maximum or mean. Second derivative values should be decreased locally to maintain the original endpoint properties which makes the degree-reduced curves flatter where second derivative changes little. Furthermore, another important conclusion derived from two tables is degree reduction can help on curve smoothing. The reason for this is the process of degree reduction reduce the number of control points which will relieve the restraint conditions at endpoints.

We consider a closed curve with degree of thirteen $(n=13)$ in the last example. From the variation of control points with different weight, the conclusion we get above is reinforced: degree-reduced curves are global smooth under the endpoint constraint (10), but local smooth under the other one. If the control points change uniformly just like the red dotted in Figure 5, the smoothing term will make an obvious effect. Otherwise the control points after degree reduction change little which is shown in Figure 6.

\section{Conclusion}

In this paper, we introduce a new approach to generate Bézier curve with an additional smoothing term based on the conventional objective function. Sometimes when the smoothness of a curve needs to be adjusted globally or locally within a smaller range, adding this term becomes required. Therefore, our objective function includes two parts: a conventional approximation error part and a smoothing part. They are organized with a weight $\omega$. The explicit representations of unknown points with conditions of two kinds of endpoint constraints are presented respectively. It is obvious from the examples that the shape of degree-reduced curve depends on three elements, namely endpoint condition, smoothing term and approximation error. Under the premise of guaranteeing endpoint condition, as the names imply, smoothing term determines the smoothness and flatness of curve while the approximation error is the main factor of proximity between degree-reduced curve and the given one. In addition, the smoothing effects are different under different endpoint constraint conditions. 


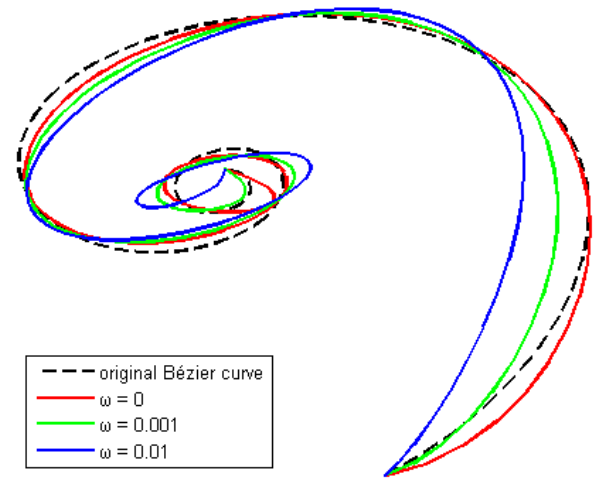

Figure 3. Degree reduced curves with constraint (10).

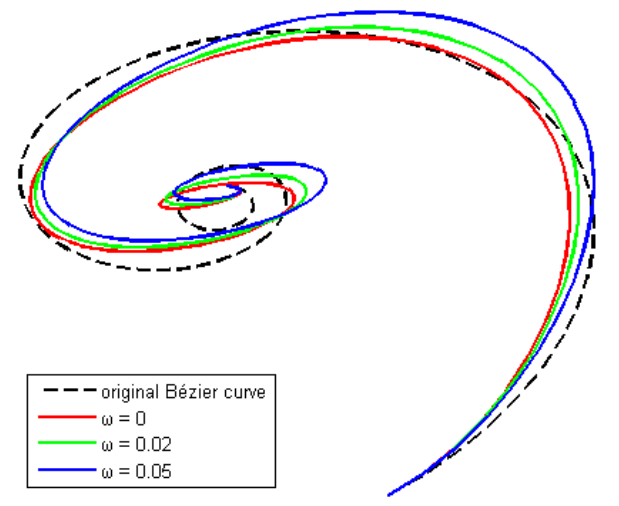

Figure 4. Degree reduced curves with constraint (13).

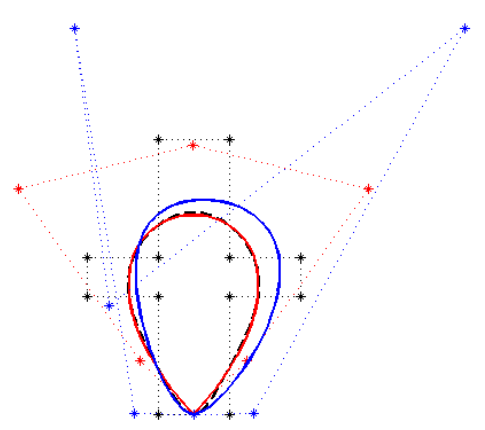

Figure 5. Degree reduced curves and control points with constraint (10).

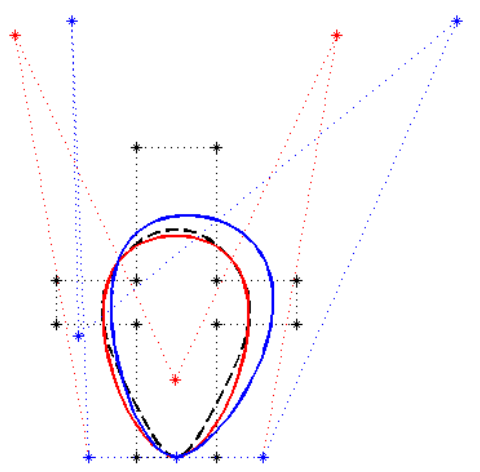

Figure 6. Degree reduced curves and control points with constraint (13). 


\section{Acknowledgements}

This work is supported by the National Natural Science Foundation of China (No. 11271376).

\section{References}

[1] Liu, L., Zhang, L., Lin, B. and Wang, G. (2009) Fast Approach for Computing Roots of Polynomials Using Cubic Clipping. Computer Aided Geometric Design, 26, 547-559. http://dx.doi.org/10.1016/j.cagd.2009.02.003

[2] Bogacki, P., Weinstein, S.E. and Xu, Y. (1995) Degree Reduction of Bézier Curves by Uniform Approximation with Endpoint Interpolation. Computer-Aided Design, 27, 651-661. http://dx.doi.org/10.1016/0010-4485(94)00011-2

[3] Eck, M. (1993) Degree Reduction of Bézier Curves. Computer Aided Geometric Design, 10, 237-251. http://dx.doi.org/10.1016/0167-8396(93)90039-6

[4] Kim, H.O. and Moon, S.Y. (1997) Degree Reduction of Bézier Curves by L ${ }^{1}$-Approximation with Endpoints Interpolation. Computers and Mathematics with Application, 33, 67-77. http://dx.doi.org/10.1016/S0898-1221(97)00020-5

[5] Rababah, A. and Mann, S. (2013) Linear Methods for $\mathrm{G}^{1}, \mathrm{G}^{2}$ and $\mathrm{G}^{3}$-Multi-Degree Reduction of Bézier Curves. Computer-Aided Design, 45, 405-414. http://dx.doi.org/10.1016/j.cad.2012.10.023

[6] Woźny, P. and Lewanowicz, S. (2009) Multi-Degree Reduction of Bézier Curves with Constraints, Using DualBernstein Basis Polynomials. Computer Aided Geometric Design, 26, 566-579.

http://dx.doi.org/10.1016/j.cagd.2009.01.006

[7] Zhou, L., Wei, Y. and Yao, Y. (2014) Optimal Multi-Degree Reduction of Bézier Curves with Geometric Constraints. Computer-Aided Design, 49, 18-27. http://dx.doi.org/10.1016/j.cad.2013.12.004

[8] Eck, M. (1995) Least Squares Degree Reduction of Bézier Curves. Computer-Aided Design, 27, 845-851. http://dx.doi.org/10.1016/0010-4485(95)00008-9

[9] Burnnett, G., Schreiber, T. and Braun, J. (1996) The Geometry of Optimal Degree Reduction of Bézier Curves. Computer Aided Geometric Design, 13, 773-788. http://dx.doi.org/10.1016/0167-8396(96)00009-X

[10] Kim, H.O., Kim, J.H. and Moon, S.Y. (1996) Degree Reduction of Bézier Curves and Filter Banks. Computers and Mathematics with Applications, 31, 23-30. http://dx.doi.org/10.1016/0898-1221(96)00049-1

[11] Sunwoo, H. (2005) Matrix Representation for Multi-Degree Reduction of Bézier Curves. Computer Aided Geometric Design, 22, 261-273. http://dx.doi.org/10.1016/j.cagd.2004.12.002

[12] Lu, L. and Wang, G. (2006) Optimal Multi-Degree Reduction of Bézier Curves with G²-Continuity. Computer Aided Geometric Design, 23, 673-683. http://dx.doi.org/10.1016/j.cagd.2006.09.002

[13] Lu, L. (2015) Some Improvements on Optimal Multi-Degree Reduction of Bézier Curves with Geometric Constraints. Computer-Aided Design, 59, 39-42. http://dx.doi.org/10.1016/j.cad.2014.08.017

[14] Gospodarczyk, P. (2015) Degree Reduction of Bézier Curves with Restricted Control Points Area. Computer-Aided Design, 62, 143-151. http://dx.doi.org/10.1016/j.cad.2014.11.009 